\title{
Posthospital follow-up visits and 30-day readmission rates in chronic obstructive pulmonary disease
}

This article was published in the following Dove Press journal:

Risk Management and Healthcare Policy

28 May 2014

Number of times this article has been viewed

\author{
Salman S Fidahussein' \\ Ivana T Croghan ${ }^{2}$ \\ Stephen S Cha ${ }^{3}$ \\ David L Klocke ${ }^{4}$ \\ 'Department of Pulmonary and \\ Critical Care Medicine, Norwalk \\ Hospital, Norwalk, CT, \\ ${ }^{2}$ Department of Medicine Clinical \\ Research Office, ${ }^{3}$ Department of \\ Health Sciences Research, ${ }^{4}$ Division \\ of Hospital Internal Medicine, \\ Department of Medicine, \\ Mayo Clinic, Rochester, \\ MN, USA
}

Purpose: To examine the effect of a follow-up visit with a primary care physician and/or pulmonologist within the first 30 days of hospital discharge on readmissions, emergency department (ED) visits, and mortality.

Patients and methods: This was a retrospective cohort study of 7,102 unique patients discharged from a Mayo Clinic hospital in Rochester, MN, and residing in Olmsted County, $\mathrm{MN}$, with any mention of chronic obstructive pulmonary disease (COPD) from January 1 , 2004 through November 30, 2011. The study included 839 patients who met study-entry criteria. Cox proportional hazards regression was performed to determine the risk of hospital readmission, ED visits, and death of patients, with or without a follow-up visit during the first 30 days postdischarge.

Results: Our results showed 839 unique patients experienced 1,422 discharges with a primary diagnosis of COPD. Of the 1,422 discharges, 973 (68.4\%) had a follow-up visit within 30 days. In a multivariate Cox proportional hazard-ratio (HR) model analysis, occurrence of a follow-up visit did not have a significant effect on the risk of the combined outcome of 30-day readmission and ED visit (HR 0.947, confidence interval 0.763-1.177; $P=0.63$ ). However, a postdischarge follow-up visit had a significant effect on 30-day mortality (HR 0.279 , confidence interval 0.149-0.523; $P<0.001)$.

Conclusion: Postdischarge follow-up visits after hospitalization for COPD did not significantly reduce the risk of 30-day readmission or ED visit. However, patients who received postdischarge follow-up visits had significantly reduced 30-day mortality.

Keywords: care transitions, COPD, discharge planning, outpatient follow-up, risk factors

\section{Introduction}

Title III of the 2010 US Patient Protection and Affordable Care Act for health care reform emphasizes reducing readmissions to improve quality and integration of care and to reduce costs for Centers for Medicare and Medicaid Services (CMS) beneficiaries. Medicare expenditures for readmissions have been estimated to be as high as $\$ 17.4$ billion a year. ${ }^{1}$ The national average for 30-day all-cause hospital readmissions is $19.3 \%(12.9 \%-26.3 \%) .{ }^{2}$ The CMS has subsequently made readmission rates a priority measure of quality, with penalties that began October 1, 2012 under the Patient Protection and Affordable Care Act and associated 2012 rule for inpatient prospective payments. ${ }^{1}$

Chronic obstructive pulmonary disease (COPD) is the fourth-leading cause of death in the US and the only disease associated with both increasing morbidity and mortality over the past 30 years. ${ }^{3}$ Resulting in 726,000 hospitalizations and 1.5 million emergency 
department (ED) visits in the US in the year $2000,{ }^{3}$ COPD has been determined to be one of the top five diagnoses for hospital readmission. ${ }^{1,4-6}$ One in five patients discharged with COPD is readmitted within 30 days postdischarge, making COPD not only common but costly. ${ }^{1,4}$ Hospital admissions due to acute exacerbations of COPD affect health-related quality of life $^{7}$ and prognosis. ${ }^{8}$ Its burden to society, as expressed in disability life-years, is predicted to rise to the fifth-leading cause worldwide for both sexes by $2020 .{ }^{9} \mathrm{CMS}$ penalties for 30-day readmissions currently target congestive heart failure, acute myocardial infarction, and pneumonia, as these are the CMS's most costly discharge diagnoses, but COPD discharges with a 30-day readmission will soon be included in the CMS's measures for readmission. ${ }^{1,10}$

The causes of readmissions are presumed to be related in part to poor coordination and quality of care, including timely postdischarge follow-up with a physician. However, evidence is lacking as to whether specific interventions, such as early follow-up, can prevent 30-day readmissions, and it is even less clear whether all diagnoses will respond similarly to any specific intervention(s). The primary aim of this study was to examine the effect of a follow-up visit with the patient's primary care physician (PCP) or a pulmonologist within the first 30 days after hospital discharge for a primary diagnosis of COPD, on readmission, ED visit, and death, collectively termed as postdischarge failure (PDF).

\section{Materials and methods}

We conducted a retrospective cohort study of patients discharged from a tertiary care academic medical center to their home or to a skilled nursing facility with a primary discharge diagnosis of COPD. Our inclusion criteria were: 1) patients had to be 18 years of age or older; 2) patients had to be discharged from a Mayo Clinic hospital in Rochester, MN, from January 1, 2004 through November 30,$2011 ; 3$ ) patients had to be residing in Olmsted County, MN; and 4) patients had to have a primary discharge diagnosis of COPD, regardless of COPD etiology (diagnosis-related group [DRG] code 088 and COPDrelated International Classification of Diseases [ICD]-9 codes 491.xx, 492.xx, 493.xx, and 496). ${ }^{6,11}$ The ICD-9 codes are consistent with National Quality Forum COPD codes. The exclusion criteria were: 1) patients who declined research authorization (as dictated by Minnesota Statute 144.335), 2) prisoners, 3) patients who expired during their hospital stay, 4) patients who left against medical advice, 5) patients who had planned readmissions using Agency for Healthcare Research and Quality (AHRQ) classifications, and (6) patients with COPD as a secondary diagnosis. The study was approved by the Mayo Clinic Institutional Review Board.

Both the predictor and outcome variables were retrieved from Mayo Clinic administrative databases. An outpatient visit to the patient's PCP or pulmonary physician within 30 days of the discharge date was defined as follow-up care for that admission. Outpatient physician visits were captured using Health Care Financing Administration current procedural terminology evaluation and management codes 99211-99220 ${ }^{12}$ (established patient encounter). PCPs and pulmonologists were flagged and recorded by the computer analyst. An important note is that all patients with discharge disposition to a nursing home are cared for by embedded Mayo Clinic PCPs, allowing full capture of administrative data on these patients. To avoid clustering of events at the patient level, we included only one admission per patient in a 30-day period for the index visit. Therefore, the next index admission date was considered for analysis if the event occurred outside the 30-day period of the last index admission. No follow-up visits after the occurrence of the primary outcome were considered, even if they occurred within the 30 days of index discharge.

Data on important confounders, such as age, hospital length of stay (LOS), and comorbidities, were also collected. ${ }^{13}$ All confounding variables with significant association were included in the multivariate analysis. To ensure data validity and integrity, every fifth random patient from a data set was verified by a manual review of medical records. If an error was found, the data-extraction program was revisited and modified until the accuracy approximated 99\%.

Assuming $20 \%$ of patients discharged with a primary diagnosis of COPD were readmitted within 30 days, and half of those patients were seen by a PCP or pulmonologist prior to that event, we estimated that a log-rank test with a two-sided significance level of 0.05 would have $80 \%$ power to detect the difference of PDF rates in 30 days between $23 \%$ and $17 \%$, ie, a hazard ratio (HR) of 0.713 for 1,400 discharges.

Patient characteristics were compared between patients with a follow-up visit and patients without a follow-up visit with either the PCP or pulmonologist within 30 days of discharge. A two-sided $t$-test was used for continuous variables, and Pearson's $\chi^{2}$ test for categorical variables. These baseline characteristics were summarized using mean ( \pm standard deviation) for continuous variables and frequency $(\%)$ for categorical variables. The HRs for follow-up visits and the corresponding 95\% confidence intervals (CIs) were estimated by a Cox proportional hazards 
model. These calculations used patients having no follow-up with their PCP or pulmonologist as a reference group. To obtain multivariate-adjusted HRs, the adjusted covariates were age, Charlson Comorbidity Index (CCI), number of COPD admissions, and index LOS. The Cox proportional hazards model was also used to identify factors associated with a follow-up visit within 30 days. The Kaplan-Meier method was used to plot the log of cumulative HRs against time for patients with and without follow-up visits. The log-rank test was used to identify the cutoff values for significant risk factors. All patients were censored after readmission, ED visit, death, and at 30 days after discharge. All reported $P$-values were two-sided, and $P<0.05$ was considered statistically significant. The data analysis was generated using SAS software version 9.3 (SAS Institute, Cary, NC, USA).

\section{Results}

Our study cohort initially consisted of 7,102 unique patients who were discharged from a Mayo Clinic hospital in Rochester, MN and resided in Olmsted County, MN from January 1, 2004 through November 30, 2011 with any mention of COPD (DRG code of 088). Of those patients, 6,041 had COPD listed as a secondary discharge diagnosis and were excluded. Sixty-six patients were excluded because they declined research authorization. Also excluded were 146 patients who did not meet the age requirement (18 years), or AHRQ readmission criteria or left against medical advice, and ten patients who died during

7,102 unique patients discharged with any mention of COPD between 01/01/2004 and 11/30/2011

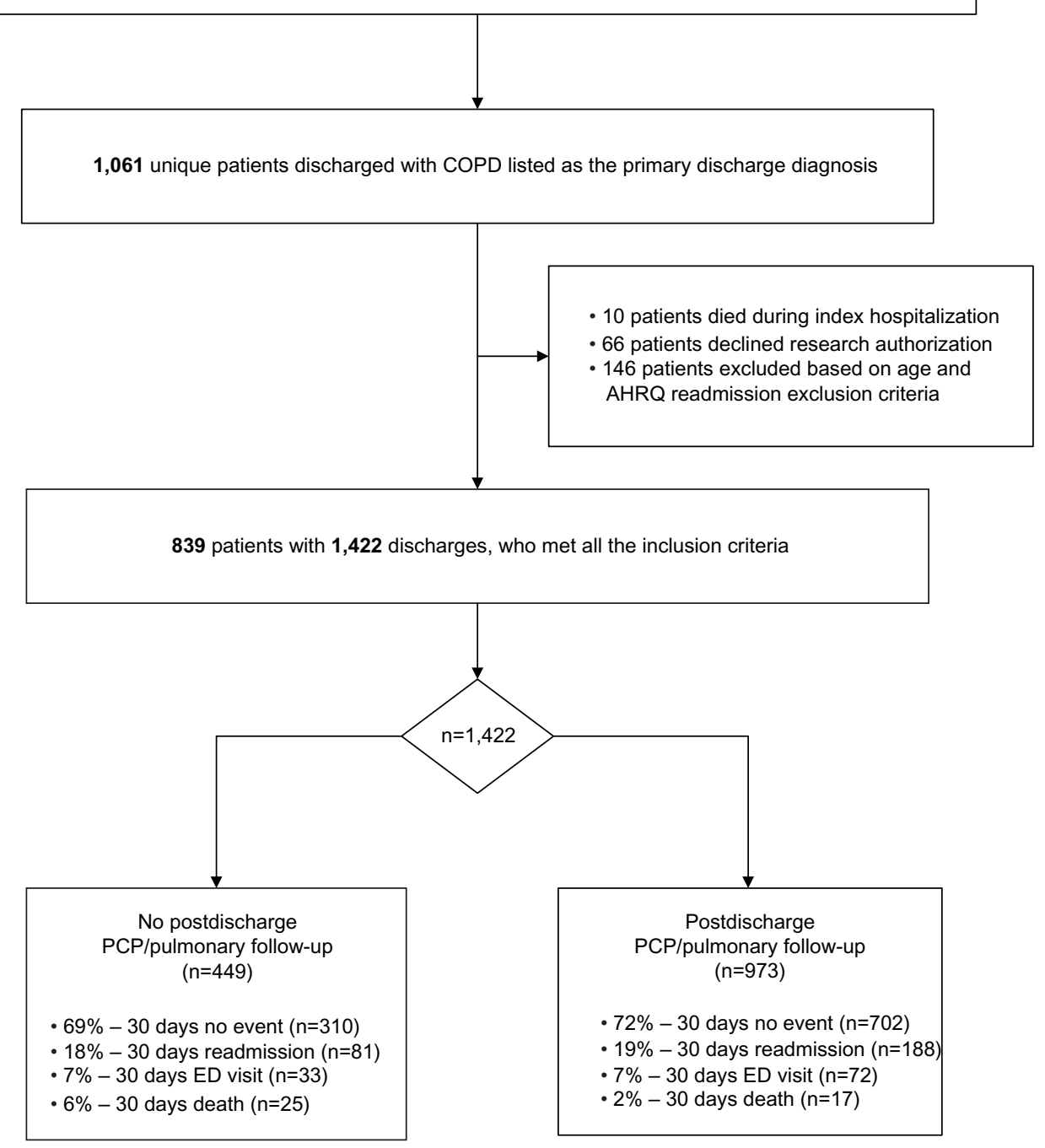

Figure I Study flow diagram of patients who met all the inclusion and exclusion criteria. 
the index admission. As seen in Figure 1, the resulting 839patient cohort that met study inclusion criteria accumulated 1,422 index discharges with a primary diagnosis of COPD. The division and percentage of patients with and without follow-up and their primary outcomes is also illustrated in Figure 1. Based on COPD DRG codes, ${ }^{6,11} 71 \%$ had a COPD-related ICD-9 code (491.xx, 492.xx, or 496) listed as the primary diagnosis. Asthma (ICD-9 code 493.xx) was listed as the primary diagnosis in an additional $29 \%$ of these admissions.

Demographics and clinical characteristics of patients with and without follow-up visits are summarized in Table 1. Between 2004 and 2011, 68\% of patients with COPD had an outpatient visit within 30 days of discharge, of whom $81 \%$ had follow-up with their PCP and 19\% had follow-up with a pulmonary specialist. The mean age of the cohort was 66 years. Women comprised $59 \%$ of the patients, and $79 \%$ of the patients were white.

The end-point outcomes are shown in Table 2. The HRs for readmission $(1.02$, CI $0.80-1.32 ; P=0.84)$ or an ED visit $(0.97$, CI $0.77-1.2 ; P=0.80)$ were not significantly different for patients who had a follow-up compared to patients who did not, after controlling for age, sex, CCI, index LOS, and cumulative admissions per patient for COPD (Figure 2A). However, a significant mortality reduction was noted with follow-up visits (HR 0.28 , CI 0.15-0.52; $P<0.001$ ) (Figure 2B). When all end points, 30-day readmissions, ED visits, or death were combined, PDF was not statistically significant between the follow-up versus the no-follow-up group (HR 0.85, CI 0.69-1.05; $P=0.13$ ) (Figure 2C).

Table I Comparison of characteristics of patients with and without a follow-up visit with their primary care physician or pulmonologist following hospitalization for COPD

\begin{tabular}{|c|c|c|c|}
\hline Characteristics & $\begin{array}{l}\text { All patients } \\
(n=1,422)\end{array}$ & $\begin{array}{l}\text { No postdischarge } \\
\text { follow-up }(n=449)\end{array}$ & $\begin{array}{l}\text { Postdischarge } \\
\text { follow-up } \\
(n=973)\end{array}$ \\
\hline Age $(n \pm S D)$ & $66 \pm 17$ & $64 \pm 18$ & $67 \pm 16$ \\
\hline LOS (days $\pm S D)$ & $2.89 \pm 3.08$ & $3.04 \pm 3.70$ & $2.82 \pm 2.75$ \\
\hline $\mathrm{CCl}(\mathrm{n} \pm \mathrm{SD})$ & $2.49 \pm 2.21$ & $2.32 \pm 2.19$ & $2.57 \pm 2.21$ \\
\hline $\begin{array}{l}\text { Cycles }(n \pm S D), \\
\text { range (min-max) }\end{array}$ & $\begin{array}{l}2.53 \pm 2.36 \\
(I-I 5)\end{array}$ & $\begin{array}{l}2.42 \pm 2.28 \\
(I-13)\end{array}$ & $\begin{array}{l}2.59 \pm 2.39 \\
(I-15)\end{array}$ \\
\hline Male sex, n (\%) & $585(4 I)$ & $187(42)$ & $398(4 I)$ \\
\hline \multicolumn{4}{|l|}{ Race, n (\%) } \\
\hline White & I, I I7 (79) & 325 (72) & $792(8 I)$ \\
\hline Black & $83(6)$ & $41(9)$ & $42(4)$ \\
\hline Others & $222(16)$ & $83(18)$ & $139(14)$ \\
\hline $\begin{array}{l}\text { English- } \\
\text { speaking, n (\%) }\end{array}$ & I,056 (74) & $327(73)$ & $729(75)$ \\
\hline
\end{tabular}

Abbreviations: SD, standard deviation; $\mathrm{CCl}$, Charlson Comorbidity Index; COPD, chronic obstructive pulmonary disease; LOS, length of stay; min, minimum; max, maximum.
We also noted that the risks for readmission or death were influenced by other factors. Table 3 summarizes the factors that were significant, as well as those that were not significant, for predicting the likelihood of readmission, ED visit, and death. For both readmissions and ED visits, only $\mathrm{CCI}$ and the number of COPD admissions were significant for predicting the likelihood of the end point. For mortality no follow-up, older age, CCI, hospital LOS, and the number of COPD readmissions were significant predictors.

\section{Discussion}

Unplanned preventable readmissions and deaths are important outcomes of hospital care, and a decrease in their frequency is an important and desirable outcome for patients and health care providers. A posthospital follow-up visit is considered by many providers as best practice, and has been suggested by some authors as a universal intervention or modifiable factor that can reduce readmission rates. Furthermore, the literature has tended to group all patients and all diagnoses in one pool, suggesting all patients and diseases might benefit equally from follow-up care. In this study, we carefully examined outcomes for COPD patients who had a follow-up visit with a pulmonologist or their PCP. We did not identify significantly different rates of 30-day readmission or 30-day posthospital ED visits in patients who attended a follow-up visit in our study cohort. However, we did find a statistically significant improvement in mortality for patients who had an outpatient follow-up visit. We do not have a good explanation for this finding, but it may be an inadvertent selection bias, with sicker patients avoiding or unable to attend follow-up visits.

The current literature on follow-up visits has shown mixed results. It is important to recognize that there are relatively few studies on this question, and most of the studies have grouped diverse diagnoses and populations together, making the assumption that follow-up care would have the same effect for all patients. Both Sharma et $\mathrm{al}^{6}$ and $\mathrm{Sin}$ et $\mathrm{al}^{14}$ reported that a follow-up visit after discharge reduced the risk of readmission and ED visits for COPD and asthma patients. On the other hand, Grafft et a ${ }^{15}$ reported that a scheduled follow-up appointment was not associated with decreased readmissions or mortality in general medicine patients. Weinberger et al ${ }^{16}$ reported that improved access to primary care following hospital discharges for older patients with diabetes, COPD, or congestive heart failure was associated with increased rates of readmissions $(P=0.005)$. Consistent with Grafft et al ${ }^{15}$ and Weinberger et al, ${ }^{16}$ this study found that readmission rates 
Table 2 Hazard ratios of 30-day hospital readmission, ED visit, death, readmission + ED visit, and postdischarge failure (readmission, ED, and death combined) for patients who had follow-up compared to patients who did not

\begin{tabular}{llllll}
\hline Outcomes & Rate $(\%)$ & Unadjusted HR (95\% Cl) & P-value & Adjusted HR $\mathbf{( 9 5 \% ~ C l )}$ & $P_{\text {-value }}$ \\
\hline Readmission & $188(19)$ & $1.07(0.83,1.37)$ & 0.59 & $1.02(0.80-1.32)$ & 0.84 \\
ED visit & $72(7)$ & $1.01(0.80,1.27)$ & 0.94 & $0.97(0.77-1.22)$ & 0.80 \\
Death & $17(2)$ & $0.31(0.17,0.57)$ & 0.001 & $0.28(0.15-0.52)$ & $<0.001$ \\
Readmission + ED visit & $260(26)$ & $0.98(0.79,1.22)$ & 0.86 & $0.95(0.76-1.18)$ & 0.63 \\
Postdischarge failure & $277(28)$ & $0.89(0.72,1.09)$ & 0.25 & $0.85(0.69-1.05)$ & 0.13 \\
\hline
\end{tabular}

Note: aAdjusted for age, sex, Charlson Comorbidity Index, length of stay, and number of admission cycles.

Abbreviations: $\mathrm{ED}$, emergency department; $\mathrm{HR}$, hazard ratio; $\mathrm{Cl}$, confidence interval.

and ED visits in patients with follow-up visits were not significantly different compared to patients without follow-up with their PCP and/or pulmonologist $(P>0.05)$.

It is important to note that the average national readmission rate is $19.3 \%$. Conversely, approximately $80.7 \%$ of patients are not readmitted. ${ }^{1}$ Therefore, applying such interventions as scheduling follow-up visits to reduce readmissions for every patient who is discharged from the hospital may not be efficient or cost-effective in our current health care environment. We reviewed a number of postdischarge-care models that identified factors predictive for early readmission in general medical patients. ${ }^{17-22}$ The literature has demonstrated that exacerbations of COPD are not random events. Instead, these admissions are clustered in time, such that there is a high-risk period for recurrence within 8 weeks after the initial exacerbation, with higher chances of readmission during this interval. ${ }^{1}$ While we did not adjust for this in our study, there is a critical need to better understand and identify readmission patterns and risk factors for common diseases if we are to effectively improve readmission rates and individualize health care interventions.

Kansagara et $\mathrm{al}^{17}$ performed a systematic review for risk-prediction models for hospital readmissions, and suggested that most current readmission risk-prediction models perform poorly for predicting readmission. Other studies have reported that having more than three COPD admissions in the year before recruitment was found to be associated with an increased risk of readmission. ${ }^{18-20}$ In our study, we identified a CCI value of greater than 3 , a hospital LOS greater than 3 days, and at least six prior admissions with a primary discharge diagnosis of COPD as risk factors predicting the likelihood of postdischarge events. We found that CCI, the absence of a follow-up visit, age, and hospital LOS contributed to predicting 30-day mortality. Ideally, application of an evidence-based "relapse risk profile" for every patient at the time of hospital admission that included CCI and other established risk factors, ${ }^{19-21}$ and accompanied by the application of proven and patient-specific interventions, could be useful for reducing readmissions while minimizing resource use. However, based on this study, it is unclear that follow-up visits are helpful for reducing readmissions for patients discharged with a primary diagnosis of COPD.

In our cohort of patients, the most common readmission diagnosis, as described by the ICD-9 coding scheme, was chronic bronchitis (ICD-9 code 491.xx), at 29.4\%. Other unspecified diseases of the lung (ICD-9 code 518.xx), heart failure (ICD-9 code 428.xx), pneumonia (ICD-9 code 486. $\mathrm{xx}$ ), and asthma (ICD-9 code 493.xx) accounted for 6.8\%, $6.3 \%, 5.7 \%$, and $5.4 \%$ of readmissions, respectively. Cumulatively, the majority of the patients discharged with a primary diagnosis of COPD were readmitted for pulmonary-related complications.

Our retrospective cohort design has several limitations. We did not identify patients who may have been readmitted or who may have had a follow-up visit with a health care provider at another institution. We mitigated this limitation by limiting our cohort to patients residing in our immediate county who would be unlikely to access health care elsewhere due to the limited options other than Mayo Clinic facilities. Any such errors would be small, and unlikely to have affected our outcomes. We excluded patients who had AHRQ exclusion diagnoses, but some of these events may have been inadvertently captured. To limit this, we manually validated multiple random samples of readmission and ED visits for DRG and ICD-9 codes.

Another possible limitation could be the quality of care during the index hospitalization. We were unable to collect data and adjust for choice of treatment, length of treatment, or adherence to treatment for each admission, which could affect outcomes. While we did not measure these variables, COPD patients at Mayo Clinic receive consistent and standardized care, with most patients admitted to a specialty pulmonary service. Also, ED and outpatient physician practice patterns can influence the decision to admit a patient independently of a patient's clinical status; 
A

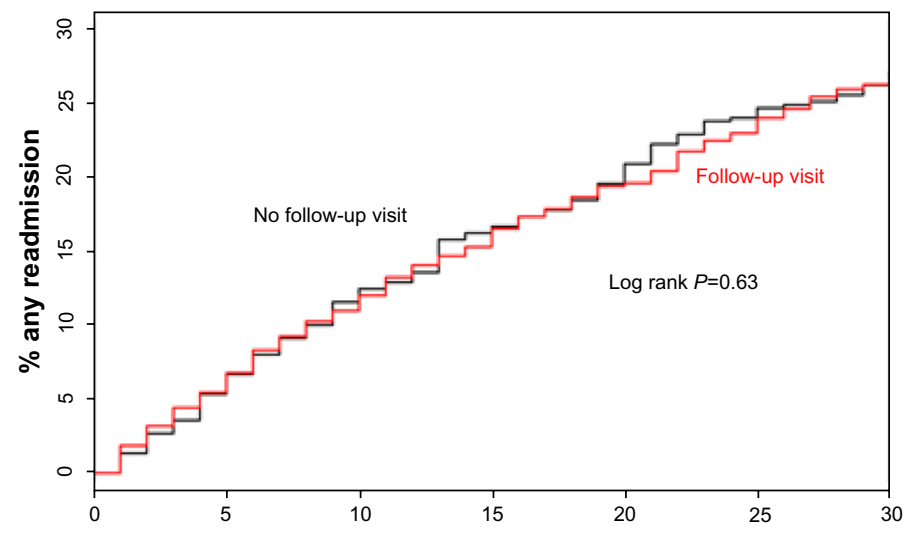

B

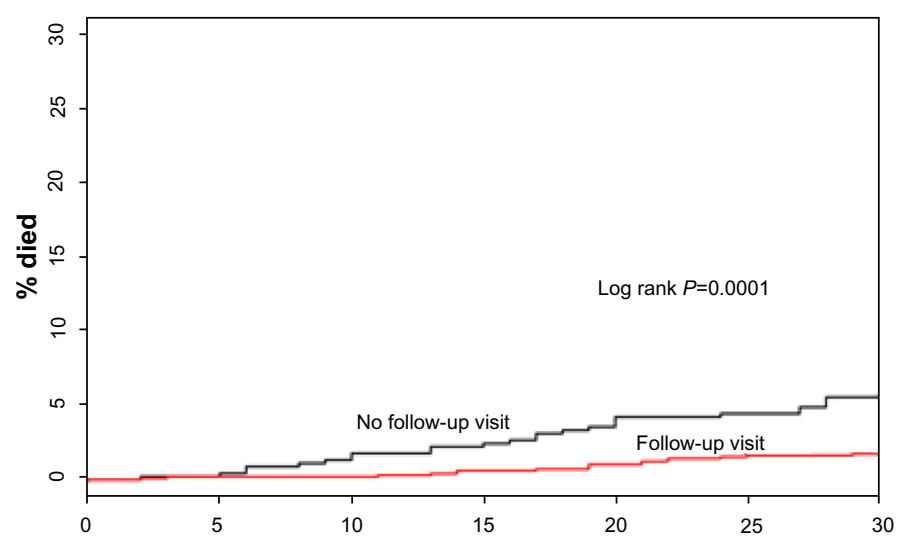

C

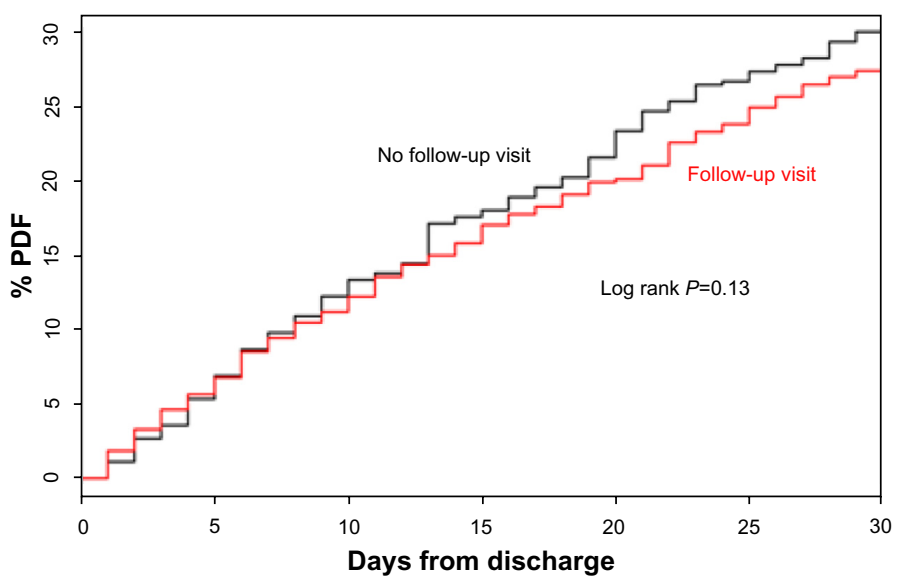

Figure 2 Cumulative rates of 30 day post-discharge events (readmission, ED visits, and post-discharge failure) for patients with and without follow-up.

Notes: (A) Cumulative rates of readmission and emergency department (ED) visits at 30 days postdischarge for patients with and without primary care physician (PCP)/ pulmonology follow-up. (B) Cumulative rates of death at 30 days postdischarge for patients with and without PCP/pulmonology follow-up. (C) Cumulative rates of postdischarge-failure (PDF) readmission, ED visit, or death at 30 days postdischarge for patients with and without PCP/pulmonology follow-up.

we did not examine this in our study, ${ }^{1}$ nor did we examine other care coordination, such as early discharge to pulmonary rehabilitation, home visits from a respiratory therapist, or nurse or self-management education. All are potentially beneficial in reducing COPD exacerbations; ${ }^{18,19}$ however, few if any such activities were occurring at Mayo Clinic during the time frame of this study.

Not adjusting for the severity of COPD is an important limitation of our study. However, we calculated CCI, incorporating factors that measured the patients' 
Table 3 Hazard ratios for risk factors of readmission, ED visit, and death within 30 days of discharge

\begin{tabular}{|c|c|c|}
\hline Risk factors & $\begin{array}{l}\text { Hazard ratio } \\
(95 \% \mathrm{Cl})\end{array}$ & $P$-value \\
\hline \multicolumn{3}{|l|}{ Readmission } \\
\hline PCP/pulmonary follow-up & $1.03(0.80-1.32)$ & 0.84 \\
\hline Age & $1.01(0.99-1.01)$ & 0.19 \\
\hline Male sex & I.I3 (0.89-I.42) & 0.32 \\
\hline Charlson Comorbidity Index & $1.13(1.09-1.17)$ & $<0.01$ \\
\hline Length of hospital stay & $1.03(0.99-1.05)$ & 0.07 \\
\hline Number of COPD admissions & $1.13(1.08-1.19)$ & $<0.01$ \\
\hline \multicolumn{3}{|l|}{ ED visit } \\
\hline PCP/pulmonary follow-up & $0.97(0.77-1.23)$ & 0.80 \\
\hline Age & $1.00(0.99-1.01)$ & 0.93 \\
\hline Male sex & $1.14(0.91-1.42)$ & 0.25 \\
\hline Charlson Comorbidity Index & $1.13(1.09-1.18)$ & $<0.01$ \\
\hline Length of hospital stay & $1.02(0.99-1.04)$ & 0.26 \\
\hline Number of COPD admissions & $1.13(1.08-1.18)$ & $<0.01$ \\
\hline \multicolumn{3}{|l|}{ Death } \\
\hline PCP/pulmonary follow-up & $0.28(0.15-0.52)$ & $<0.01$ \\
\hline Age & $1.06(1.03-1.09)$ & $<0.01$ \\
\hline Male sex & $1.24(0.67-2.31)$ & 0.50 \\
\hline Charlson Comorbidity Index & $1.15(1.05-1.26)$ & $<0.01$ \\
\hline Length of hospital stay & $1.06(1.03-1.10)$ & $<0.01$ \\
\hline Number of COPD admissions & $1.21(1.06-1.38)$ & $<0.01$ \\
\hline
\end{tabular}

Abbreviations: $\mathrm{Cl}$, confidence interval; COPD, chronic obstructive pulmonary disease; ED, emergency department; PCP, primary care physician.

overall state of health. Socioeconomic status, a possible confounder, was not considered. The apparent mortality benefit may have been related to selection bias, and merits further study

Using binary variables (yes/no), measurement precision was not an issue, and increasing the sample size could have further decreased the likelihood of random error. Our power statement demonstrated the ability of our study to detect significant differences between groups.

We believe that the results from our study are valid for the population and setting we studied (a single center in the Midwest region of the US). Further study is required to provide validation of these results for all COPD patients and in other settings. In determining the value of follow-up visits, it is important to establish which, if any, outcomes can be affected, especially since many patients in the US do not have ready access to follow-up care. In addition, none of the studies we are aware of, including this one, explored the content and process of a posthospital follow-up visit. Physicians are more likely to focus on symptoms and treatment when other factors, such as the ability to afford and properly take medications and treatment, as well as patient education about triggers for treatment escalation, could be more appropriate activities at follow-up.

\section{Conclusion}

Postdischarge follow-up did not significantly reduce 30-day readmissions, ED visits, or the combined measure of PDF, but postdischarge follow-up did have a statistically significant effect on the mortality of patients with COPD. Identifying and reducing avoidable readmissions has the potential to improve patient safety and satisfaction, enhance quality of care, and reduce health care spending. Further study is necessary regarding the benefit of follow-up for other diseases. If, in contradiction to these results, follow-up visits are eventually demonstrated to be effective for reducing readmissions for COPD patients, it is likely that such follow-up visits would need to be scripted and standardized to specifically achieve that aim. CMS began penalizing hospitals with higher rates of readmission for acute myocardial infarction, congestive heart failure, and pneumonia on October 1, 2012, and will expand penalties to other diagnoses, including COPD, in 2015. Therefore, it is urgent that we develop a better understanding of what factors identify at-risk patients and what interventions might reduce the risk of readmission for subpopulations of patients, in order to improve care and to direct health care resources effectively.

\section{Acknowledgment}

The authors are grateful for support from the Mayo Clinic Department of Internal Medicine Clinical Research Office.

\section{Disclosure}

The authors report no conflicts of interest in this work.

\section{References}

1. Jencks SF, Williams MV, Coleman EA. Rehospitalizations among patients in the Medicare fee-for-service program. $N$ Engl J Med. 2009;360(14):1418-1428.

2. Oelschlaeger A. Geographic variation in readmissions and potentially avoidable hospitalizations. Poster presented at: Academy Health Annual Research Meeting: Advancing Research, Policy and Practice; June 12, 2011; Seattle, WA.

3. Centers for Disease Control and Prevention (CDC). Deaths from chronic obstructive pulmonary disease - United States, 2000-2005. MMWR Morb Mortal Wkly Rep. 2008;57(45):1229-1232.

4. Camberg LC, Smith NE, Beaudet M, Daley J, Cagan M, Thibault G Discharge destination and repeat hospitalizations. Med Care. 1997;35(8) 756-767.

5. Koekkoek D, Bayley KB, Brown A, Rustvold DL. Hospitalists assess the causes of early hospital readmissions. J Hosp Med. 2011;6(7): 383-388.

6. Sharma G, Kuo YF, Freeman JL, Zhang DD, Goodwin JS. Outpatient follow-up visit and 30-day emergency department visit and readmission in patients hospitalized for chronic obstructive pulmonary disease. Arch Intern Med. 2010;170(18):1664-1670.

7. Seemungal TA, Donaldson GC, Paul EA, Bestall JC, Jeffries DJ, Wedzicha JA. Effect of exacerbation on quality of life in patients with chronic obstructive pulmonary disease. Am J Respir Crit Care Med. 1998;157(5 Pt 1):1418-1422. 
8. Mannino DM. COPD: epidemiology, prevalence, morbidity and mortality, and disease heterogeneity. Chest. 2002;121(Suppl 5):121S-126S.

9. Murray CJ, Lopez AD. Alternative projections of mortality and disability by cause 1990-2020: Global Burden of Disease Study. Lancet. 1997;349(9064):1498-1504.

10. Medicare Payment Advisory Commission public meeting [transcript]. 2011. Available from: http://www.medpac.gov/transcripts/1215162011. pdf. Accessed August 7, 2013.

11. Commission on Professional and Hospital Activities. The International Classification of Diseases, Ninth Revision, Clinical Modification: ICD-9-CM. Ninth Revision. Ann Arbor (MI): Commission on Professional and Hospital Activities; 1992.

12. American Medical Association. CPT Professional Codebook: CPT 2015 Professional Edition. Chicago: American Medical Association; 2015.

13. Charlson ME, Pompei P, Ales KL, MacKenzie CR. A new method of classifying prognostic comorbidity in longitudinal studies: development and validation. J Chronic Dis. 1987;40(5):373-383.

14. Sin DD, Bell NR, Svenson LW, Man SF. The impact of follow-up physician visits on emergency readmissions for patients with asthma and chronic obstructive pulmonary disease: a population-based study. Am J Med. 2002;112(2):120-125.

15. Grafft CA, McDonald FS, Ruud KL, Liesinger JT, Johnson MG, Naessens JM. Effect of hospital follow-up appointment on clinical event outcomes and mortality. Arch Intern Med. 2010;170(11):955-960.
16. Weinberger M, Oddone EZ, Henderson WG. Does increased access to primary care reduce hospital readmissions? Veterans Affairs Cooperative Study Group on Primary Care and Hospital Readmission. N Engl J Med. 1996;334(22):1441-1447.

17. Kansagara D, Englander H, Salanitro A, et al. Risk prediction models for hospital readmission: a systematic review. JAMA. 2011;306(15): 1688-1698.

18. Connolly MJ, Lowe D, Anstey K, Hosker HS, Pearson MG, Roberts CM. Admissions to hospital with exacerbations of chronic obstructive pulmonary disease: effect of age related factors and service organisation. Thorax. 2006;61(10):843-848.

19. Garcia-Aymerich J, Barreiro E, Farrero E, Marrades RM, Morera J, Antó JM. Patients hospitalized for COPD have a high prevalence of modifiable risk factors for exacerbation (EFRAM study). Eur Respir J. 2000;16(6):1037-1042.

20. Garcia-Aymerich J, Monso E, Marrades RM, et al. Risk factors for hospitalization for a chronic obstructive pulmonary disease exacerbation. EFRAM study. Am J Respir Crit Care Med. 2001;164(6):1002-1007.

21. Bahadori K, FitzGerald JM. Risk factors of hospitalization and readmission of patients with COPD exacerbation - systematic review. Int J Chron Obstruct Pulmon Dis. 2007;2(3):241-251.

22. Bourbeau J, Julien M, Maltais F, et al. Reduction of hospital utilization in patients with chronic obstructive pulmonary disease: a disease-specific self-management intervention. Arch Intern Med. 2003;163(5): 585-591.
Risk Management and Healthcare Policy

\section{Publish your work in this journal}

Risk Management and Healthcare Policy is an international, peerreviewed, open access journal focusing on all aspects of public health, policy, and preventative measures to promote good health and improve morbidity and mortality in the population. The journal welcomes submitted papers covering original research, basic science, clinical \& epidemio-

\section{Dovepress}

logical studies, reviews and evaluations, guidelines, expert opinion and commentary, case reports and extended reports. The manuscript management system is completely online and includes a very quick and fair peerreview system, which is all easy to use. Visit http://www.dovepress.com/ testimonials.php to read real quotes from published authors. 